\title{
On the Evaluation of Texture and Color Features for Nondestructive Corrosion Detection
}

\author{
Fátima N. S. Medeiros, ${ }^{1}$ Geraldo L. B. Ramalho, ${ }^{2}$ Mariana P. Bento, ${ }^{1}$ \\ and Luiz C. L. Medeiros ${ }^{3}$ \\ ${ }^{1}$ Teleinformatics Engineering Department, Federal University of Ceara, Campus of Pici, 6007 Fortaleza, CE, Brazil \\ ${ }^{2}$ Federal Institute of Education, Science, and Technology of Ceara, Campus of Maracanau, Av. Contorno Norte 10, \\ 61925-315 Maracanau, CE, Brazil \\ ${ }^{3}$ Petroleo Brasileiro S.A., LUBNOR/IE, Av. Leite Barbosa s/n, 60180-420 Fortaleza, CE, Brazil \\ Correspondence should be addressed to Fátima N. S. Medeiros, fsombra@deti.ufc.br
}

Received 2 January 2010; Accepted 15 June 2010

Academic Editor: João Marcos A. Rebello

Copyright () 2010 Fátima N. S. Medeiros et al. This is an open access article distributed under the Creative Commons Attribution License, which permits unrestricted use, distribution, and reproduction in any medium, provided the original work is properly cited.

\begin{abstract}
We present a methodology for automatic corrosion detection in digital images of carbon steel storage tanks and pipelines from a petroleum refinery. The database consists of optical digital images taken from equipments exposed to marine atmosphere during their operational life. This new approach focuses on color and texture descriptors to accomplish corroded and noncorroded surface area discrimination. The performance of the proposed corrosion descriptors is evaluated by using Fisher linear discriminant analysis (FLDA). This approach presents two main advantages: No refinery stoppages are required and potential-related catastrophes can be prevented.
\end{abstract}

\section{Introduction}

Corrosion is the destructive attack of a metal by chemical or electrochemical reaction with its environment [1]. The exposure of metallic surfaces structures to rust degradation during their operational life is a known problem and it affects storage tanks, steel bridges, pipelines, and ships [2].

Storage tanks and pipelines are commonly made of carbon steel and low alloy steel not resistant to corrosion in natural environments. Corrosion resistant paints and coatings are used in almost all applications. Despite of this protection, some steels will rust quite rapidly in humid air even though condensation is not evident [3].

Atmospheric corrosion causes economic losses usually due to production interruptions, replacement of expensive materials, and contamination of products. A good deal of attention should be paid to safety risks and the environment pollution due to corrosion [4]. Therefore, corrosion monitoring is a relevant task to detect corroded regions before failures occur by using inspection methods, so that appropriate decision-making can be taken to avoid any untoward incidents.

There are different corrosion analysis methods as mechanical measurements, chemical analysis, and visual inspection by experts. Inspection usually refers to evaluation of attributes in relation to a specification. For economical and security reasons, the petroleum and gas industry requires nonintrusive or nondestructive measurement techniques to avoid disturbing their properties and performance [5].

Visual inspection of metallic surface is a common practice employed to identify and detect sources of failures. A tough problem associated with this task is its tedious and subjective nature. Significant refinement can be achieved if specialists have access to automatic computerized inspection methods.

In many materials, the corrosion process produces a typical rough surface. Therefore, texture analysis is highly recommended to discriminate specific surface roughness. Texture is defined as repetition of a pattern over a region as a set of small variations, generally described by a spatial 
function [6]. Another issue is the random aspect of texture, because size, shape, and orientation of pattern elements can vary over a region.

The potentiality of image processing techniques for automatic rust steel detection is investigated in [2]. The methodology introduced an iterative multivariate data analysis to examine the effects of rust steel descriptors, that is, texture and color distribution on a set of classifier algorithms. In this analysis, a selector of classifiers indicates the algorithm that provides good classification results (high sensitivity) and acceptable time response for the automation of the system [2].

In 1981, Itzhak et al. [7] employed computer image processing techniques for statistical evaluation of pitting corrosion in a plate of AISI 304L stainless steel exposed to a corrosive water solution containing $10 \% \mathrm{FeCl}_{3}$. The purpose of this work was to introduce and to evaluate new tools for analyzing the effects of pitting corrosion process [7]. The algorithm was capable of estimating the number and area of pits in the binary image and therefore provided better evaluation of pitting corrosion damages.

A popular image processing algorithm for texture analysis extracts features from the gray level co-occurrence matrix (GLCM) [8]. In this paper, we explore the power of these features to deal with the stochastic pattern of corrosion for damage detection in metallic surfaces. Parameters extracted from the GLCM can be used to define similarity properties for corrosion detection purpose in image segmentation methods based on region approach. This approach consists in determining the regions that contain neighbor pixels in the image that have similar properties, that is, gray level and spatial relationship [9]. Two GLCM parameters, namely contrast and energy, are considered to be the most efficient for discriminating different textural patterns [10].

A wide variety of literature works $[6,8,10,11]$ have reported that texture features are proper to characterize corroded surfaces. In addition, typical color changes of metallic surfaces are often related to corrosion. Thus, color attributes carry out relevant information to design corrosion detection systems. Moreover, some works have reported that feature combination carries more discriminant power to applications designed on small database image samples [12]. Methods based on neural networks and feature selection are able to handle with high data dimensionality maintaining good generalization level $[13,14]$.

This paper proposes a robust feature set for reliable detection of atmospheric corrosion on metallic surfaces using optical images acquired by charge-coupled device (CCD) cameras. A total of 13 attributes per image sample were computed using color and texture models: HSI (hue, saturation, and intensity) color histogram statistics and GLCM probabilities. The GLCM probabilities measure the roughness, and HSI statistics characterize the color of metallic surface samples. A sequential bottom-up feature selection procedure [15] was applied as a result of the small sample size and the high data dimensionality. We use Fisher linear discriminant analysis (FLDA) and receiver operating characteristic (ROC) curve to investigate the performance of

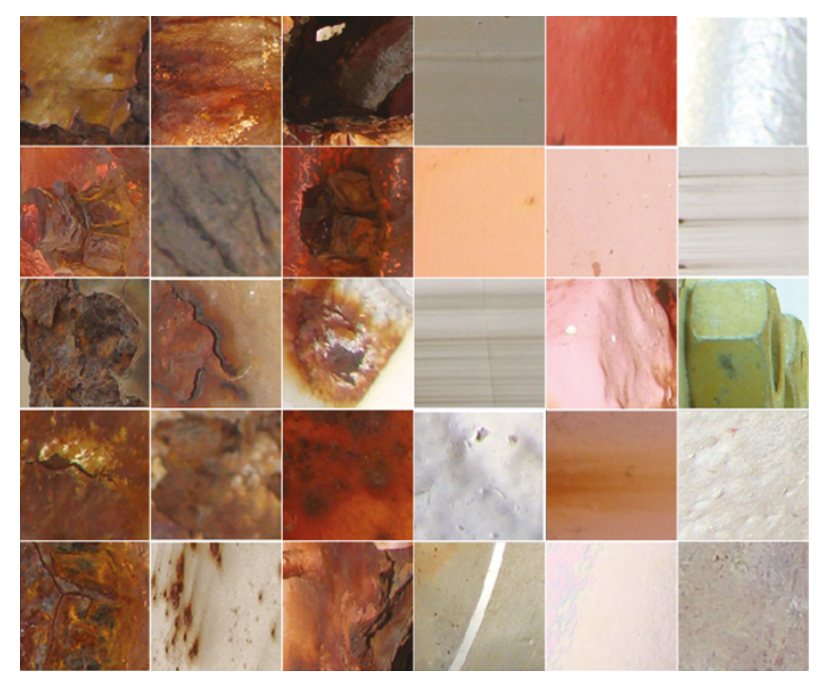

FIgURE 1: Image database. The three columns on the left illustrate metallic surfaces attacked by atmospheric corrosion. The three columns on the right illustrate a variety of smooth and rough non corroded surfaces.

the proposed approach when combining texture and color feature subsets.

The outline of the paper is as follows. In the next section, we describe the image database and give a brief overview of texture and color attributes used for corrosion characterization. Section 3 introduces the proposed methodology and aspects of feature sets evaluation. The experimental results and performance analysis are covered in Section 4. In Section 5, we draw the concluding remarks.

\section{Materials and Methods}

2.1. Image Database. A set of 33 high-resolution images was collected from storage tanks of a petroleum plant. The images were obtained under different acquisition conditions of illumination and magnification. Some images show a large number of corrosion defects, while others give a detailed view of a single defect. An expert selected 84 regions of interest (ROI), each one resulting $128 \times 128$ pixels small images containing true corrosion, corrosion-like and noncorrosion samples. A subset of 43 ROI images represent different corrosion damages. The remaining 41 ROI images contain non corroded surfaces or corroded-like surfaces. Figure 1 illustrates the image database.

2.2. Texture Attributes. Texture is formally defined as the set of local neighborhood properties of the gray levels of an image region [16]. It reaches intuitive attributes as roughness, granulation, and regularity. There are four different methods for texture analysis in the literature: statistical, structural, model-based and transform-based methods.

The gray level intensity distribution of an image is based on the assumption that texture information is contained in the spatial relationship between the intensities of a pixel and its neighbor [8]. This information is condensed in the 
GLCM. The gray level intensity distribution can be specified by a matrix of relative frequencies, in which two neighbor elements of texture labeled $i$ and $j$, separated by a distance $d$ in an orientation $q$, occur in the image, one with property $i$ and other with property $j$.

GLCM encompasses at least 14 texture attributes [8]. Although, for simplicity sake, we adopt an optimized subset of 4 attributes that is, contrast, correlation, energy, and homogeneity [10] given by

$$
\begin{aligned}
\text { Contrast } & =\sum_{k} k^{2} \sum_{i} \sum_{j} S(i, j), \\
\text { Correlation } & =\sum_{i, j} \frac{i j S(i, j)-\mu_{i} \mu_{j}}{\sigma_{i} \sigma_{j}}, \\
\text { Energy } & =\sum_{i, j} S^{2}(i, j), \\
\text { Homogeneity } & =\sum_{i, j} \frac{S(i, j)}{1+(i-j)^{2}} .
\end{aligned}
$$

The matrix $S$ represents GLCM and the sum index $k$ in (1) is denoted by GLCM size minus one less one. The parameters $\mu_{i}, \mu_{j}, \sigma_{i}$, and $\sigma_{j}$ in (2) represent, respectively, the mean value and standard deviation of line $i$ and column $j$ from GLCM.

Contrast measures the dissimilarity intensity between a pixel and its neighbor over the whole image. Correlation represents how a pixel is related to its neighbor over the whole image. Energy is the sum of squared elements in GLCM, also known as uniformity of energy. Homogeneity stands for the similarity between gray level values of image pixels.

Homogeneity and contrast identify organized structures in the image. Energy and correlation characterize the complexity and nature of gray level transitions in the image. Even though these attributes contain information about image texture, it is difficult to identify which specific texture characteristic is represented by each attribute. Hence, texture attributes are stored in a feature database for further characterization by a classification process.

2.3. Color Attributes. Color is the visual perception of the spectral distribution of the light. Optical imaging uses three color channels, usually associated with red $(R)$, green $(G)$, and blue $(B)$, sufficient for the visual interpretation of spectra [16]. In applications of image corrosion detection by using digital image processing and pattern recognition algorithms, it is relevant to identify the best color model to represent color attributes.

The HSI system constitutes a model that best describes how humans naturally respond to color. Thus, the HSI color space is appropriate for this purpose since it allows describing characteristics separately from brightness chrominance [11].
The hue, saturation, and intensity are obtained from RGB color space by using the following transformations:

$$
\begin{gathered}
H=\frac{R+G+B}{3}, \\
S=1-\frac{3}{R+G+B}[\min (R, G, B)], \\
I=\cos ^{-1} \frac{[(R-G)+(R-B)] / 2}{\sqrt{(R-G)^{2}+(R-B)(G-B)}} .
\end{gathered}
$$

Hue $(H)$ is proportional to the color frequency as (5) describes. For a corroded surface, $H$ lies between yellow and red wavelengths.

Saturation $(S)$ refers to the dominance of hue in the color and is given by (6). A corroded surface is normally more saturated than other areas because metallic surface is often painted in light colors as gray and white.

Intensity ( $I$ ) is given by (7) and describes the strength of the light. As explained before, the color of non corroded surface tends to white wavelength (high intensity).

Color attributes are obtained by using statistical moments extracted from each HSI channel histogram. We adopted the histogram definition as a frequency $x_{n}$ for each pixel value, where $n=1 \ldots N$ and it refers to imaging quantization.

Each statistical moment provides a different meaning. Furthermore, the first moment (8) indicates where the individual color generally lies in the HSI color space. The second moment (9) incorporates the information on the spread or scale of the color distribution. Non corroded surfaces are often homogeneous and they imply low variance. The third moment (10) measures the asymmetry of the data around the sample mean and indicates when the HSI values lie toward maximum or minimum in the scale. The fourth moment (11) measures the flatness or peakedness of the color distribution as follows:

$$
\begin{gathered}
E(X)=\frac{1}{N} \sum_{n=1}^{N} x_{n}, \\
E\left((X-E(X))^{2}\right)=\frac{1}{N} \sum_{n=1}^{N}\left(x_{n}-E(X)\right)^{2}, \\
E\left((X-E(X))^{3}\right)=\frac{1}{N} \sum_{n=1}^{N}\left(x_{n}-E(X)\right)^{3}, \\
E\left((X-E(X))^{4}\right)=\frac{1}{N} \sum_{n=1}^{N}\left(x_{n}-E(X)\right)^{4} .
\end{gathered}
$$

\section{Methodology for Corrosion Characterization}

3.1. Corrosion Descriptors. Automatic corrosion damage detection on metallic surfaces is a complex task which requires a multistep procedure. Figure 2 summarizes the feature extraction step used to design our proposed NDE (nondestructive evaluation) system. In our approach, we perform a discriminant analysis based on digital image features encompassing texture and color. 


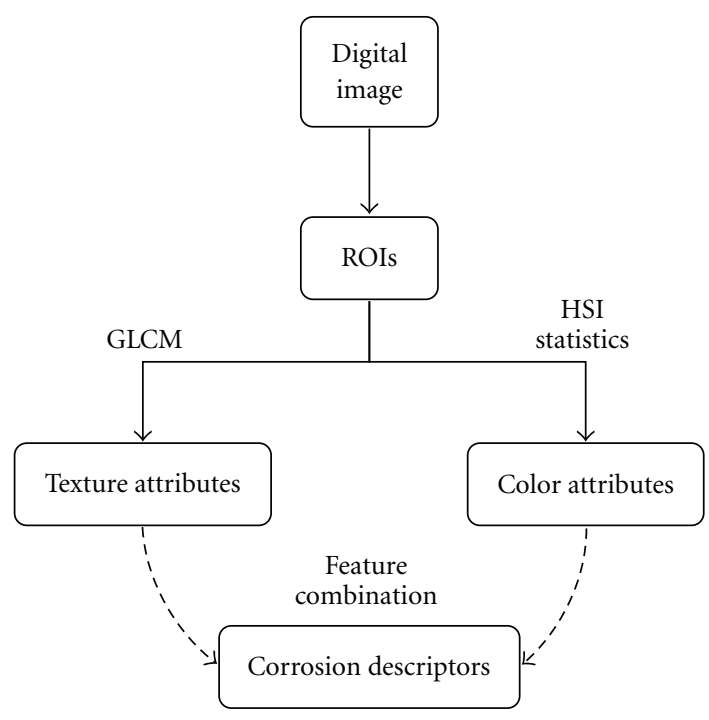

FIGURE 2: Overview of the corrosion descriptors design.

We propose a corrosion descriptor database organized into three-feature subsets: texture attributes, color attributes and a combination of texture and color attributes. GLCM has been computed by assigning the distance between pixels to 1 and orientation of neighbor pixels to $90^{\circ}$. Attributes defined in (8)-(11) have been calculated for the histogram of the hue, saturation, and intensity components of the HSI color space.

3.2. Discriminant Analysis. Figure 3 illustrates the discriminant analysis of attributes to identify corroded and non corroded surfaces. The principal component analysis (PCA) is a mathematical procedure that optimizes the feature set by eliminating redundant attributes. The result is a smaller number of uncorrelated attributes called principal components.

Fisher Linear Discriminant Analysis (FLDA) [17] is applied to the three-feature subsets in order to compare the attributes robustness for corrosion detection. The discriminant fits a multivariate normal density to each data group, with a pooled estimate of covariance $\mathbf{S}_{W}$ defined by

$$
\mathbf{S}_{W}=\frac{1}{n-2}\left(n_{1} \Sigma_{1}+n_{2} \Sigma_{2}\right)
$$

which maximizes the criterion

$$
J=\frac{\left|\mathbf{w}^{T}\left(\mathbf{m}_{1}-\mathbf{m}_{2}\right)\right|^{2}}{\mathbf{w}^{T} \mathbf{S}_{W} \mathbf{w}},
$$

where $\mathbf{m}_{i}$ is the mean and $n_{i}$ the sample size of class $\omega_{i}$, $i=1 \cdots 2 ; \Sigma_{i}$ is the maximum likelihood estimates of the covariances matrices of classes $\omega_{i}$; w represents the new space that maximizes the criterion. According to (13), the criterion proposed by Fisher is the ratio of between-class to withinclass variances.

The regression $\mathbf{w}^{T} \mathbf{x}+w_{0}=0$ obtained by FLDA is used to assess the discrimination performance for each feature subset. The corroded class and non corroded class separability is investigated for different subset sizes.
TABLE 1: Confusion Matrix for corrosion characterization based on texture features.

\begin{tabular}{lccc}
\hline & & \multicolumn{2}{c}{ Real } \\
\hline \multirow{3}{*}{ Predicted } & Corroded & Non corroded \\
& Non corroded & 0.4167 & 0.1071 \\
& 0.0953 & 0.3809 \\
\hline
\end{tabular}

Confusion matrix and receiver operating characteristic (ROC) curve are used to evaluate the class separability, robustness, and reliability. This matrix consists of two rows and two columns that report the number of true negatives, false positives, false negatives, and true positives estimated by the model comparing with the previous probabilities of the validation data. True values refer to correct model estimation, while false values correspond to incorrect estimated results. Error rate is quantified by the sum of false values, while accuracy is the sum of true values.

The confusion matrix provides the general discriminant performance for each feature subset. In order to determine the more appropriated subset, we used a receiver operating characteristic (ROC) curve analysis [17] to estimate the expected performance of a discriminant function under varying criterion. Sensitivity, specificity, and area under curve (AUC) are properties used to assess the performance for different number of attributes. The area under ROC Curve (AUC) gauges the ranking of correct class separation. When dealing with a reduced database sample, the area under ROC convex hull ( $\mathrm{ROCCH}$ ) provides the finest grade of the separability for the corrosion classes [17].

\section{Experimental Results}

We performed both qualitative and quantitative discriminant analysis in order to evaluate the effectiveness of different feature subsets separability.

A set of 13 attributes per image sample were computed from color and texture models using HSI statistics and gray level co-occurrence matrices (GLCMs) probabilities.

The feature subsets discriminant performance was compared in terms of classification error and execution time on a 13-dimensional (two feature subsets), 2-class ( $\omega_{1}$ and $\left.\omega_{2}\right)$ image database. For sake of simplicity, the two classconditional densities were admittedly Gaussian-like, with mean vectors $\mathbf{m}_{1}$ and $\mathbf{m}_{2}$ and a pooled covariance matrix $\mathbf{S}_{W}$ defined in (12).

The experimental results are summarized in Tables 1-4 and Figures 4, 5 and 6. The correct class discrimination for texture feature is computed as the sum of the highlighted values in the main diagonal of the confusion matrices. The values in Tables 1 and 2 reveal that about 79\% hits were obtained for texture features as much as for color features. The false negatives and false positives rates are of the same order $(\backsim 20 \%)$ for both subsets.

Combining feature sets leads to obtain more discriminant power without loss of generality. We achieved over $90 \%$ hits and an appreciably reduction of false positives to 


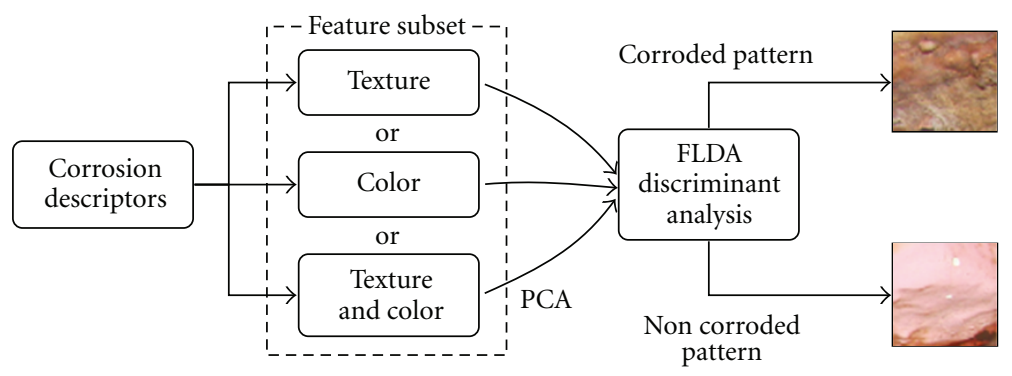

FIgURE 3: Proposed corrosion detection methodology.

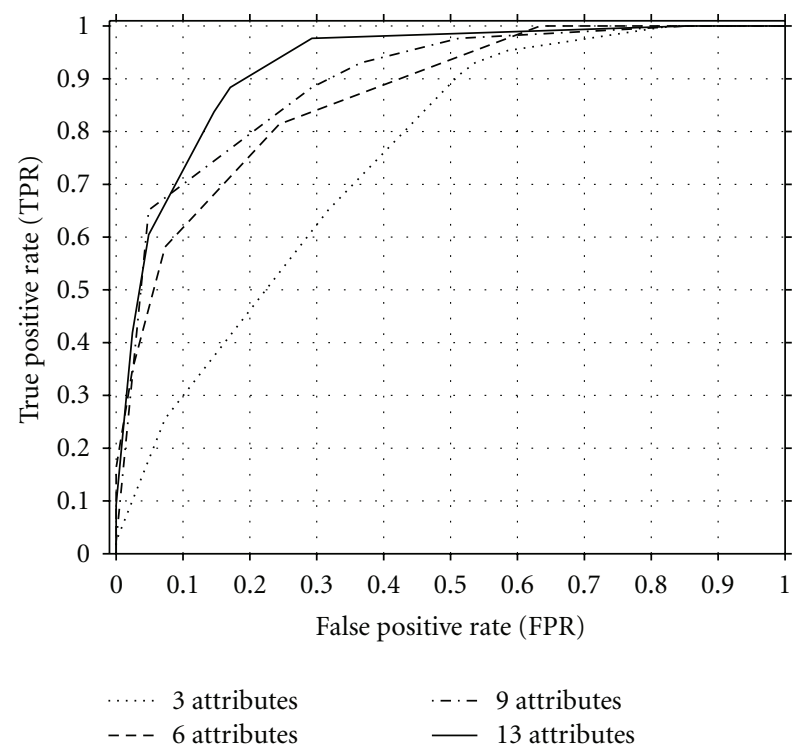

FIGURE 4: ROC convex hulls for different sizes of feature set.

TABLE 2: Confusion Matrix for corrosion characterization based on color features.

\begin{tabular}{lccc}
\hline & & \multicolumn{2}{c}{ Real } \\
\hline \multirow{3}{*}{ Predicted } & Corroded & 0.3809 & Non corroded \\
& Non corroded & 0.1309 & 0.0833 \\
& &
\end{tabular}

TABle 3: Confusion Matrix for corrosion characterization based on texture and color features.

\begin{tabular}{lccc}
\hline & & \multicolumn{2}{c}{ Real } \\
\hline \multirow{3}{*}{ Predicted } & Corroded & 0.4118 & Non corroded \\
& Non corroded & 0.0393 & 0.0392 \\
& &
\end{tabular}

less than $4 \%$. The combination of texture and color features reduced by $10 \%$ the number of false negatives and false positives. The values are reported in Table 3.

In Figure 4, we compare the ROC convex hulls obtained for different numbers of attributes. We noticed that the discriminant function aggregates more separability power as the feature set grows.

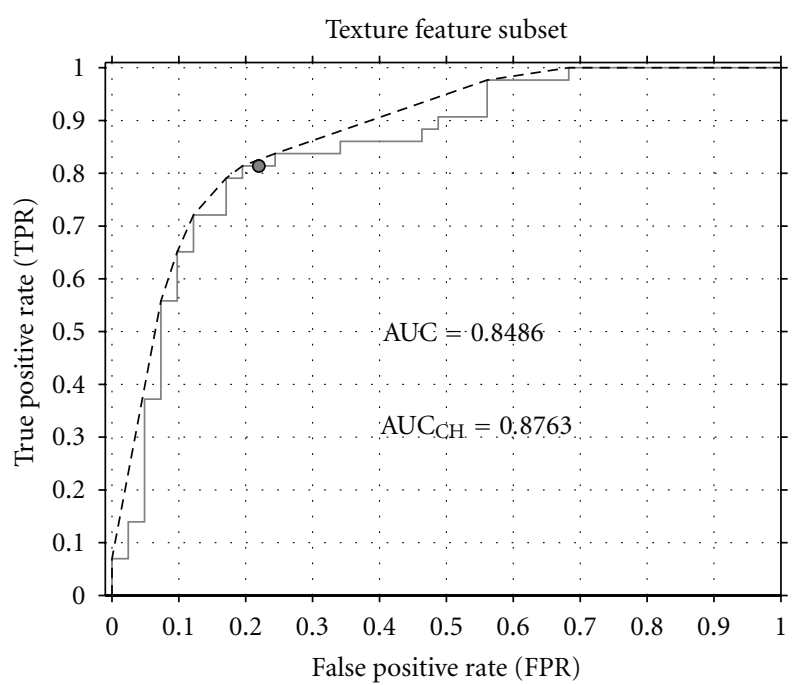

FIGURE 5: ROC analysis for corrosion characterization based on texture features. The black dot over the curve represents poor specificity and sensitivity. The solid line represents the ROC graph and the dashed line the convex hull.

The ROC curves in Figures 5-7 show that satisfactory results were achieved when texture and color features are combined. The ROC curve presents graphically the statistics estimates of false positive and true positive corrosion detection rates. We have observed from the tests that the false positive rate (FPR) is minimized, while the true positive rate (TPR) is maximized when providing texture and color attributes, thus aggregating more discriminant information. This effect is noticed by the black dot position probabilistically representing the specificity (1-true negative rate) and the sensitivity (true positive rate) for a given feature subset. The AUC evidences whether a subset is more separable than the other regardless of the relative costs of misclassification. Table 4 gives the values for AUC and $\mathrm{AUC}_{\mathrm{CH}}$. The separability of the best feature set is highlighted in gray.

The methodology was prototyped in a well-known numerical mathematics scripting environment. The execution time of the scripts is reported in terms of processor ticks spent on a $2.66 \mathrm{GHz}$ Core 2 Duo processor. Ten randomly generated feature subsets, each with 84 image patterns, were tested and the averages of the runs are reported. The 


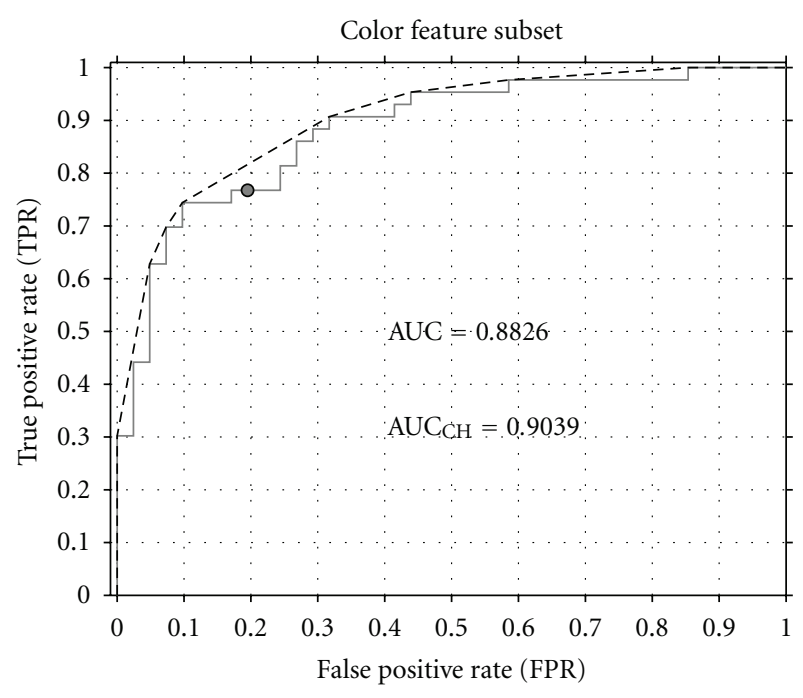

FIGURE 6: ROC analysis for corrosion characterization based on color features. The black dot represents modest specificity and sensitivity. The dashed line indicates an ROC convex hull.

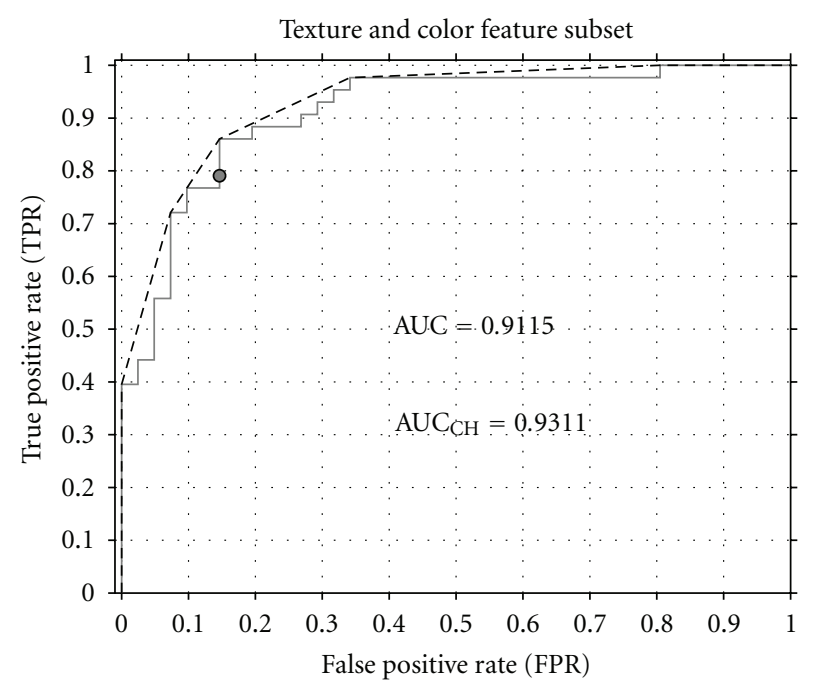

FIgURE 7: ROC analysis for corrosion characterization based on joint texture and color features. The black dot close to the upperleft corner indicates high specificity and sensitivity. The dashed line indicates an ROC convex hull.

TABle 4: Area Under Curve comparison for each feature subset.

\begin{tabular}{lcc}
\hline Feature subset & AUC & AUC $_{\mathrm{CH}}$ \\
\hline Texture & 0.8486 & 0.8763 \\
Color & 0.8826 & 0.9039 \\
Texture and Color & 0.9115 & 0.9311 \\
\hline
\end{tabular}

attributes are sequentially added one by one starting with the color subset. In the following the texture attributes are added until complete all 13 available attributes. Figure 8 shows that the error rate decays as the dimensionality increases more discriminant information. Although the execution time has an increase tendency with dimensionality of the feature set,

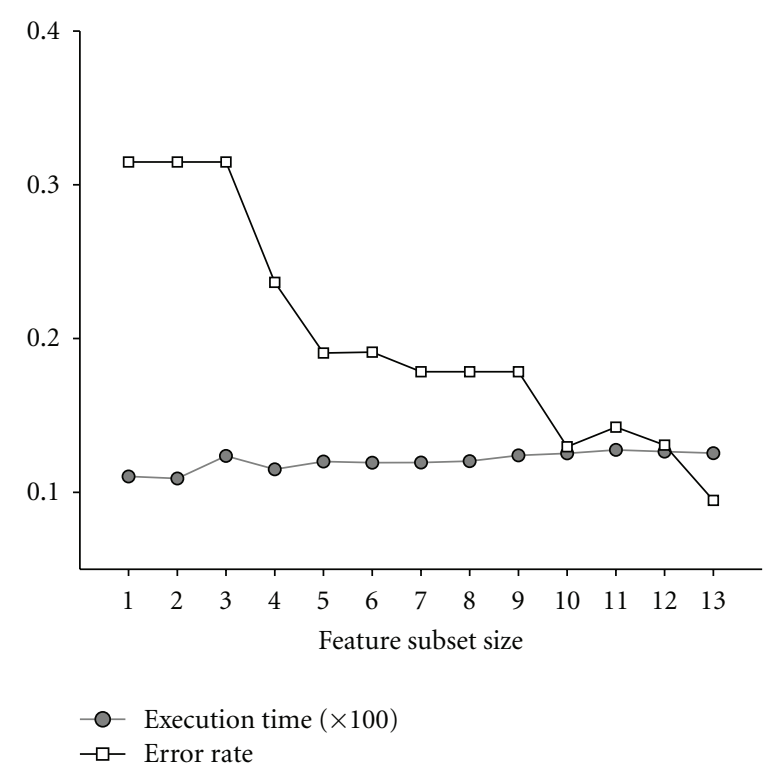

FIGURE 8: Performance evaluation for different feature subset sizes.

it is noteworthy the decreasing error rate (from 30\% to 10\%) when using a larger number of attributes.

\section{Conclusion}

In this paper, we have investigated image texture and color descriptors with the objective to address nondestructive atmospheric corrosion detection on petroleum plant equipments exposed to marine atmosphere. The idea of developing computer systems to assist specialists in corrosion inspection is to provide a tool to prevent risks for human life and environment in addition to minimizing economic losses.

Our approach integrates texture and color features to describe roughness and typical color changes in metallic surfaces. Thus, to address corrosion description, GLCM probabilities and HSI color space statistics are extracted from optical images of metallic surfaces, regardless of light and variations. Although texture and color feature subsets are likely to characterize corrosion individually, we demonstrated that when combining both of these subsets with a suboptimal sequential feature selection procedure the combination outperformed each one, that is, subset. Moreover, a linear discriminant analysis revealed that it minimized false positives and false negatives in corrosion detection.

Further work will investigate these corrosion descriptors for image segmentation in atmospheric corrosion detection systems.

\section{Acknowledgment}

The authors would like to thank ANP PRH31 for providing financial assistance. 


\section{References}

[1] J. William and D. Callister, Fundamentals of Materials Science and Engineering, John Wiley \& Sons, New York, NY, USA, 5th edition, 2001.

[2] M. Trujillo and M. Sadki, "Sensitivity analysis for texture models applied to rust steel classification," in Electronic Imaging Science and Technology, vol. 5303 of Proceedings of SPIE, pp. 161-169, San Jose, Calif, USA, January 2004.

[3] M. Kutz, Handbook of Environmental Degradation of Materials, William Andrew, NewYork, NY, USA, 2007.

[4] E. Bardal, Corrosion and Protection, Springer, Berlin, Germany, 2003.

[5] P. R. Roberge, Corrosion Inspection and Monitoring, John Wiley \& Sons, New York, NY, USA, 2007.

[6] A. S. Arko Lucieer and P. Fisher, "Multivariate texture-based segmentationof remotely sensed imagery for extraction of objects and theiruncertainly," International Journal of Remote Sensing, vol. 2917-2936, pp. 610-621, 2005.

[7] D. Itzhak, I. Dinstein, and T. Zilberberg, "Pitting corrosion evaluation by computer image processing," Corrosion Science, vol. 21, no. 1, pp. 17-22, 1981.

[8] K. S. Robert, R. M. Haralick, and I. Dinstein, "Textural features for image classification," IEEE Transactions on Systems, Man and Cybernetics, vol. 3, no. 6, pp. 610-621, 1973.

[9] S. Chabrier, B. Emile, C. Rosenberger, and H. Laurent, "Unsupervised performance evaluation of image segmentation," EURASIP Journal on Applied Signal Processing, vol. 2006, Article ID 96306, pp. 1-12, 2006.

[10] A. Baraldi and F. Parmiggiani, "An investigation of the textural characteristics associated with gray level cooccurrence matrix statistical parameters," IEEE Transactions on Geoscience and Remote Sensing, vol. 33, no. 2, pp. 293-304, 1995.

[11] K. Y. Choi and S. S. Kim, "Morphological analysis and classification of types of surface corrosion damage by digital image processing," Corrosion Science, vol. 47, no. 1, pp. 1-15, 2005.

[12] D. F. A. Lopes, G. L. B. Ramalho, F. N. S. de Medeiros, R. C. S. Costa, and R. T. S. Araújo, "Combining features to improve oil spill classification in SAR images," in Structural, Syntactic, and Statistical Pattern Recognition, vol. 4109 of Lecture Notes in Computer Science, pp. 928-936, Springer, Berlin, Germany, 2006.

[13] G. L. B. Ramalho and F. N. S. Medeiros, "Using boosting to improve oil spill detection in SAR images," in Proceedings of the 18th International Conference on Pattern Recognition (ICPR '06), pp. 1066-1069, August 2006.

[14] G. L. B. Ramalho and F. N. S. de Medeiros, "Improving reliability of oilspill detection systems using boosting for highlevel feature selection," in Proceedings of the International Conference on Image Analysis and Recognition, Lecture Notes in Computer Science No. 4633, pp. 1172-1181, August 2007.

[15] A. Jain and D. Zongker, "Feature selection: evaluation, application, and small sample performance," IEEE Transactions on Pattern Analysis and Machine Intelligence, vol. 19, no. 2, pp. 153-158, 1997.

[16] S. Livens, Image analysis for material characterisation, Ph.D. dissertation, Instelling Antwerpen University, 1988.

[17] A. R. Webb, Statistical Pattern Recognition, John Wiley \& Sons, London, UK, 2nd edition, 2002. 